\title{
Accounting Professionals, Earnings Judgments, and Tone: Based on the Undergraduate Educational Perspective
}

\author{
Chia-Hsin Cheng ${ }^{1, a}$, Chia-Ching Chang ${ }^{2, b,}{ }^{,}$, and Mao-Chao Wang ${ }^{3, c}$ \\ 1, 3Department of Accounting, Wenzhou Business College \\ Wenzhou, Zhejiang Province, 325035, China \\ ${ }^{2}$ Department of Business Administration, China University of Science and Technology \\ Taipei, Taiwan Province, 11581, China \\ a1811129827@qq.com, bsusan@cc.cust.edu.tw, c419780299@qq.com \\ ${ }^{*}$ Corresponding author
}

Keywords: Accounting Professionals, Earnings Judgments, Tone.

\begin{abstract}
To reduce the investor's confusion from the financial reports, the key point is to explain the manager's perception embedded by tone and by accounting professionals. This mission should be put into the goal of undergraduate education. Thus, this paper empirically disclosures how the accounting educationand the career encouragement could improve the financial judgments of Chinese undergraduate students, and denotes that the accounting trainings in undergraduate level might make contribution to clear financial misunderstanding.
\end{abstract}

\author{
会计专业、财报理解与文本意涵 \\ 一一基于高等院校本科教育的视角 \\ 郑家兴 $1, \mathrm{a}$, 张佳菁 $2, \mathrm{~b},{ }^{*}$, 王茂超 $3, \mathrm{c}$ \\ 1,3温州商学院会计学院, 浙江温州, 325035, 中国 \\ 2中华科技大学企业管理研究所，台湾台北，11581，中国 \\ a1811129827@qq.com, bsusan@cc.cust.edu.tw, c419780299@qq.com \\ *通讯作者
}

关键词:会计专业，财报理解，文本意涵

中文摘要.大学培训的会计专业人员, 是否能够正确理解企业财务报告中透漏的经营前景, 是 减少高管人员利用文意表述影响投资者理解的重要关键之一。本文通过实证表明, 高校会计 专业人才的培养越是扎实, 对未来专业生涯的鼓励越是加强, 学生对财务报表的理解就越清 晰明确，受文本表述中对情绪影响就越小，从而确立了会计教育对社会的重要性。

\section{1. 引言}

财务报告可以反映公司的财务状况，经营成果和现金流量等信息。因此，投资者可以利 用财务报告来了解企业运营的前景，以作出经济决策。我国会计准则明确规定，财务报告的 目标是在满足投资者在考虑决策时的信息需求。但由于公司高级管理人员的机会主义和自身 利益考虑, 表现于财务报告内文中的操弄表述, 可能造成投资者错误的估计。因此, 有许多 不可靠的信息，在没有会计人员专业判断的情况下，会造成社会无谓的损失。 
本文的研究动机是探索大学会计本科的专业训练, 是否使学生具备识别财务信息内涵之 专业能力。会计人员具备有效和更好地解读财报的专业知识和能力, 才能在社会上发挥传递 正确信息和监督企业营运的作用。因此，在高校的商业管理课程中，会计专页课程的训练是 非常重要的。中国高校会计专业本科专业, 在经过多年的改革调整后, 已具备一定的专业培 养能力, 因而促进会计本科生数量的加速增长。但大多数投资者除了会计专业知识缺乏外, 还容易受到蕴含在财报内容表达的文本意涵影响，从而做出错误的投资判断。例如在披露公 司信息时，使用积极正向的语言可能使投资者对公司未来营运有更高的评价，同时提高公司 对投资者的吸引力 ${ }^{(1)}$ 。由于会计本科生是经过严格商管专业训练培养出来的, 因此文本意涵 是否也会影响他们的专业判断，值得探讨。

本文的实证结果发现，会计本科生的专业训练有助于对公司营运前景有清晰的认识，但 专业训练的成效不应仅由学术成就来衡量。此外, 会计专业训练有助于减少文本意义的干扰。 而大学生过去的学习经历, 相关商业管理知识的充足性, 及未来从事会计工作的意愿都会影 响他们对财报的理解程度。因此, 在十九大优先发展教育事业的倡导下, 本文建议大学教师 需重视学生过去的学习经验, 借由正向情绪带动学生思考学习, 并启发学生就其本科专业上, 与其未来前途产生连结与进步的动力。

\section{2. 文献综述}

人们在语义表达上，似乎本身就存在正向与负向的意涵，及对未来的看法。因此，企业财 务报告在表述上，总有难以被会计准则约束的空间，为法律当局及投资大众所困扰。其中， Elliott ${ }^{(2)}$ 首次指出企业高管当局会利用模糊不清的会计语义表达，来左右投资人对企业前景 的判断。后续学者开始以文本分析（textual analysis）来研究高管当局到底在财报里强调了哪 些意涵 ${ }^{(3)}$ ，也有学者着重在分析方法的应用 ${ }^{(4)}$ ，信息披露的形式 ${ }^{(1)}$ ，或在不同的产业中有 哪些文本表达方式。而随着大数据与网络体系的形成, 信息阅读者背景的复杂, 以各种数据 挖掘的方式来获取高管当局报道消息的意图, 变得越来越困难 ${ }^{(5)}$ 。

由于会计专业人才的极度缺乏，及对社会责任的使命，要从教育立场来解释财务报告的语 意理解, 并不容易 ${ }^{(6)}$ 。不过, 从我国高等会计教育对文本表达的重视来看, 越来越多的学者 从国内外比较中发现, 我国所需要的会计人才, 不仅要能较好的执行会计准则的要求, 尚能 有较强的职业判断能力 ${ }^{(7)}$ 。因此, 开展理论与应用型结合的本科会计高等教育实践教学, 配 合互联网+的大数据财务信息分析, 以产生足够的决策判断力, 成为训练会计本科专业人才重 要的挑战 ${ }^{(8)}$ 。

综上所述，如何提升会计本科学生对财报信息内涵有足够的敏感度，以理解企业高管当局 背后所表述的语意, 从而做出正确的判断, 是目前我国会计本科教育需要填补的地方。过去 文献如Tan et al. (2014) ${ }^{(9)}$ 均证实企业高管当局的语调, 对企业未来的经营有预测效果, 但并 未考虑会计本科专业训练的影响力。本文试图弥补此一不足, 假设高校会计本科学生的专业 程度, 会影响阅读财报后对企业的前景理解。且所受的专业训练越高, 越不容易被财报内容 中强烈文本意涵影响。因此将本科专业人才的培育成果，合并财报里的文本意涵，对会计人 员的职业判断作一实证性的解释, 相信能约束高管当局表达夸大与不实的行为, 健全市场信 息监督，并提供会计专业更多可发挥的空间与前途。 


\section{3. 研究设计}

\section{1 问卷设计与样本}

本文问卷分两部分：第一部分测试会计本科学生面对财报个案的反应，而第二部分则调 查学生学习与生活环境的基本背景。问卷包含四份个案, 包括高科技个案 2 份, 制造业 1 份及 金融业1份, 以确保个案所属产业的完整性。此外, 在受测学生未知的情形下, 四份个案分别 设计成正向情绪的好消息，中性情绪的好消息，负向情绪的坏消息及中性情绪的坏消息，以 掌握文本意涵对阅读者的影响。正式问卷施测共有396位学生作答, 得到有效样本 1,422 组。 其中好消息共706组（包含正向情绪388组和负向情绪318组），坏消息共716组（包含负向情 绪360组及中性情绪356组）。

\section{2 文本意涵的衡量}

为确保个案具有文本意涵的代表性, 本文根据Tan et al.(2014)对文本意涵的检定方式, 将内 容的强烈情绪与中性情绪差异, 进行假设检定。实证结果如【图1】所示。学生在面对正向情 绪的好消息时，平均回馈值（平均值为 2.29）比中性情绪的好消息（平均值为 2.58 ）低; 而在 面对负向情绪的坏消息（平均值为3.24）比中性情绪的坏消息（平均值为2.94）高，印证了行 为财务学里关于股价震撼 (shock) 的影响远高过惊喜 (surprise) 的影响, 也显示本科生对财 报的消息好坏有不同的偏好。

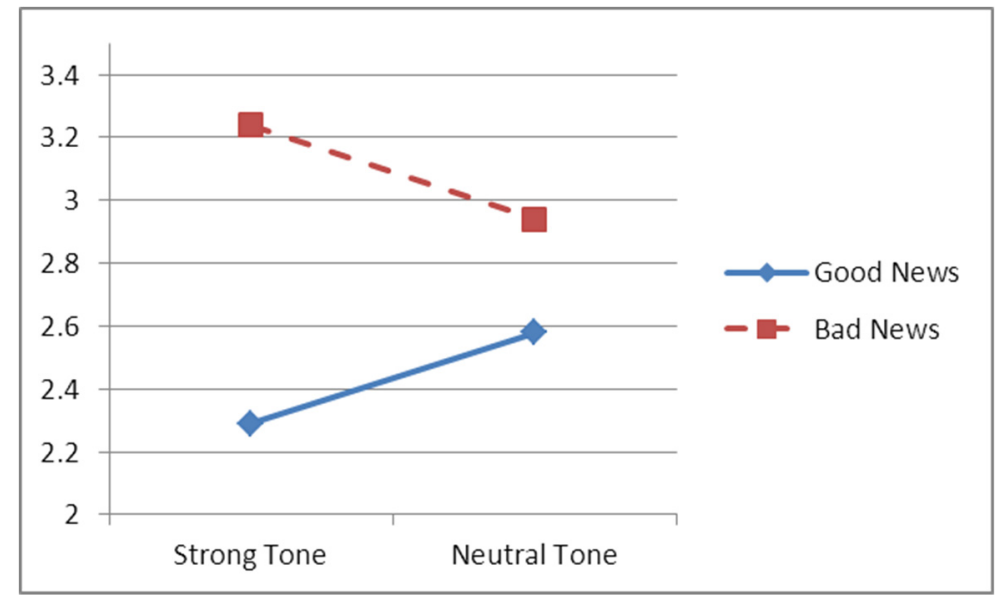

图1 会计本科生对财报文本意涵的不同反应

\section{3 实证变量}

本文汇总主要变量的内涵界定和测度方法, 如【表1】所示, 并依此建构实证模型。其中 Jud代表本科生理解反应的高低, Pro与Scr分别代表学生主观自我认定与客观专业成绩认定的 专业能力。另加上已具备之基础商学知识（Basic），过去的学习体验（Exper），及未来投入 会计事业的意愿 $(\mathrm{Fu})$ 作为控制变数, 目的是掌握住本科学生在受测当下的与教育相关的主 要因素, 及可能干扰学习背景的因素。 
表1 主要变量代号及说明

\begin{tabular}{|c|c|c|c|}
\hline 变量属性 & 变量名称 & 变量定义 & 测度方法 (李克特氏五点量表) \\
\hline 因变量 & Jud & 对公司前景的理解 & $\begin{array}{l}\text { 完全理解 }=4 ; \\
\text { 完全不理解 }=0 ;\end{array}$ \\
\hline \multirow[t]{2}{*}{ 自变量 } & Pro & $\begin{array}{l}\text { 学生自我认为的 } \\
\text { 专业程度 }\end{array}$ & $\begin{array}{l}\text { 程度佳 }=5 ; \\
\text { 程度差 }=1 ;\end{array}$ \\
\hline & Scr & 学生本科期末成绩 & 学期期末考成绩除以 10 。 \\
\hline 调节变量 & Tone & $\begin{array}{l}\text { 财报内容置入的 } \\
\text { 文本意涵 }\end{array}$ & $\begin{array}{l}\text { 哑变量, } \\
\text { 强烈情绪=1; 中性情绪=0; }\end{array}$ \\
\hline \multirow[t]{3}{*}{ 控制变量 } & Basic & 其他专业知识 & 程度佳 $=5$; 程度差 $=1$ ； \\
\hline & Exper & 过去学习经验 & $\begin{array}{l}\text { 非常良好 }=2 \text {; 非常不佳 }=-2 \text {; } \\
\text { 普通 }=0\end{array}$ \\
\hline & $\mathrm{Fu}$ & 未来投入意愿 & $\begin{array}{l}\text { 与会计完全相关 }=2 \\
\text { 与会计完全不相关 }=-2 \\
\text { 看就业机会决定 }=0\end{array}$ \\
\hline
\end{tabular}

\section{4. 实证讨论与结论}

由于问卷数据是间段性整数，不符合简单最小二乘回归的假设，因此，本文采用广义线 性模型(generalized linear model, GLM)进行模型实证分析。实证结果如下：

$$
\begin{array}{r}
\text { Jud }=1.99+0.22 * \text { Pro }-0.11 * \text { Tone } \\
\text { Jud }=2.61+0.01 * \text { Scr }-0.07 * \text { Tone } \\
\text { Jud }=0.84+0.93 * \text { Pro }-0.15 * \text { Tone }+0.20 * \text { Pro } * \text { Tone }+0.86 * \text { Basic }+0.04 * \text { Exper }+0.02 * \text { Fu } \\
\text { Jud }=0.05+0.24 * \text { Scr }-0.11 * \text { Tone }+0.02 * \text { Scr } * \text { Tone }+0.76 * \text { Basic }+0.06 * \text { Exper }+0.02 * \text { Fu }
\end{array}
$$

对照公式(1)及公式(2)的单变量分析, 学生主观的专业训练能力(Pro)显着性均较客观的学期 成绩(Scr)为高, 显示若仅以学业成绩衡量本科生专业程度略嫌不足; 而管理层在财报表述的 文本意涵(Tone), 在四种模型中均为显着负值, 显示本科生在接受会计专业训练后, 较不受 管理层表述中带有的情绪所误导。此外, 控制变数对财报理解的影响, 均为正值, 显示其对 于财报理解的正向影响。

在十九大优先发展教育事业的倡导下，本文在教育方面凸显高校会计本科的专业教育，确 实有助于财报使用者避免被管理当局用强烈的文本意涵所误导。此外, 启示会计专业教学需 重视学生过去学习经验, 与未来的事业发展。最后, 本文认为会计基础教育的社会化及普及 化, 不仅有助于减少部分投资人对上市企业的错误了解, 而且利于约束企业高管当局对信息 的做假操弄。因此，本文对于我国会计本科教育的人才培育成果，有着指标性的贡献。 


\section{References}

[1] J. Zhang, C. He, and D, Han, The Disclosure Formats of Negative CSR Information, the Linguistic Tone of Explanations and Investor's Judgments: An Experimental Research, Nankai Business Review, vol. 19, pp. 133-140, 2016.

[2] W. B. Elliott, Are Investors Influenced by Pro Forma Emphasis and Reconciliations in Earnings Announcements? The Accounting Review, vol. 81, pp. 113-133, 2006.

[3] T. I. M. Loughran, and B. McDonald, When Is a Liability Not a Liability? Textual Analysis, Dictionaries, and 10-Ks, Journal of Finance, vol. 66, pp. 35-65. 2011.

[4] P. M. Tetlock, S.Tsechansky, and S. Macskassy, More than words: Quantifying language to measure firms' fundamentals, Journal of Finance, vol. 63, pp. 1437-1467, 2008.

[5] E. Henry, and A. J. Leone, Measuring Qualitative Information in Capital Markets Research: Comparison of Alternative Methodologies to Measure Disclosure Tone, The Accounting Review, vol. 91, pp. 153-178, 2016.

[6] R. G. Benjamin, Reconstructing readability: Recent developments and recommendations in the analysis of text difficulty. Educational Psychology Review, vol. 24, pp. 63-88, 2012.

[7] K. T. Wang, and X. M. Hu, Analysis of qualitative structure of high quality accounting talents, China Higher Education, vol. 9, pp. 57-59, 2017.

[8] H. C. Duan, Research on Practice Teaching of Accounting Major in Application: Oriented Colleges, Heilongjiang Researches on Higher Education, vol. 268, pp. 165-167, 2016.

[9] H. T. Tan, E. Y. Wang, and B. Zhou, When the Use of Positive Language Backfires: The Joint Effect of Tone, Readability, and Investor Sophistication on Earnings Judgments, Journal of Accounting Research, vol. 52, pp. 273-302, 2014. 